\title{
1600 actuator tweeter mirror upgrade for the Palomar Adaptive Optics system (PALAO)
}

Richard G. Dekany, Mitchell Troy, Gary L. Brack, Charles A. Bleau, Raymond C. DuVarney, et al.

Richard G. Dekany, Mitchell Troy, Gary L. Brack, Charles A. Bleau, Raymond C. DuVarney, Mark A. Ealey, "1600 actuator tweeter mirror upgrade for the Palomar Adaptive Optics system (PALAO)," Proc. SPIE 4007, Adaptive Optical Systems Technology, (7 July 2000); doi: 10.1117/12.390293 


\title{
1600 actuator tweeter mirror upgrade for the Palomar Adaptive Optics System (PALAO)
}

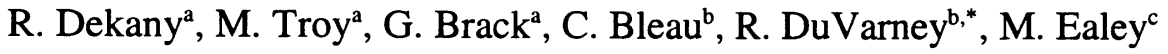 \\ aJet Propulsion Laboratory, California Institute of Technology, Pasadena, CA 91109

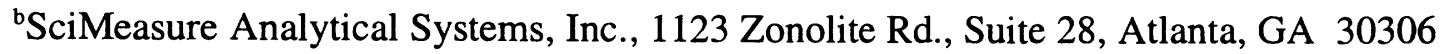 \\ 'Xinetics, Inc., 410 Great Road \#A6, Littleton, MA 01460
}

\begin{abstract}
We discuss conceptual design issues for a 1600 actuator tweeter mirror / multiconjugate AO upgrade to the 349 actuator Palomar Adaptive Optics System (PALAO). Based upon a 42 x 42 actuator Photonex deformable mirror technology, developed by Xinetics, Inc., this upgrade would enable unique science at visible wavelengths and deliver unprecedented near-infrared Strehl ratios for modestly bright $(\mathrm{mV}=9)$ guide stars. When used in conjunction with the existing 349 actuator Xinetics, Inc. deformable mirror, a series of pressing issues regarding the practical utility of multiconjugate adaptive correction for extremely large telescopes could be addressed. By utilizing a low noise (EEV39) wavefront sensor camera developed by SciMeasure Analytical Systems, Inc., this system would provide on-axis K-band Strehl ratio of $>95 \%$, improving scientific throughput and enabling the detection and spectroscopy of unresolved companions in an unprecedented contrast space around nearby stars.
\end{abstract}

Keywords: Deformable mirror, multiconjugate adaptive optics, wavefront sensing

\section{INTRODUCTION}

The demands of a new generation of extremely large telescopes (ELTs) will require new approaches to wavefront management, including the implementation of hierarchical and multiconjugate wavefront control strategies. The goal of correcting for atmosphere-induced distortions to the diffraction-limit over wide science fields calls for creative, new thinking on how to wavefront sense and correct the three-dimensional structure of the atmospheric turbulence. Multiconjugate adaptive optics (MCAO) is a promising concept ${ }^{1}$, but requires considerable new theory, followed by experimental investigation to determine its practical limitations.

Several of the outstanding questions for MCAO include:

- How many deformable mirrors are needed to correct a certain field of view (FOV) to a certain level of uniformity?

- How many guide stars are required to determine the vertical distribution of turbulence to a required fidelity?

- To what extent can open loop (non-compensated) guide star information be exploited in the turbulence reconstruction?

- To what extent can laser guide star (LGS) power requirements be minimized by exploiting nearly every natural guide star (NGS) photon?

The development of wide-field multiconjugate adaptive optics systems also requires the implementation of multiple deformable mirrors in cascade. With each mirror designed to correct for the amplitude, frequency, and spatial scale of errors likely to occur at its conjugate altitude in the atmosphere, AO system designer find a new set of design tools, for which there is little practical experience in the astronomical community. We describe below a MCAO/high-order AO testbed suited to the determination of these practical questions as they may impact the development of adaptive optics capability for CELT ${ }^{2}$.

Recent advances in high-density deformable mirror technology by Xinetics, Inc., allow us to consider an unprecedented upgrade to PALAO with the addition of a 1600 actuator deformable mirror that would be dedicated to the correction very rapid, high spatial frequency wavefront errors in the atmosphere, as well as static high spatial frequency errors in the

In Adaptive Optical Systems Technology, Peter L. Wizinowich, Editor,

Proceedings of SPIE Vol. $4007(2000) \bullet 0277-786 \times / 00 / \$ 15.00$ 
telescope primary mirror. The conceptual testbed described below would be made cost-effective by utilizing our existing 349 actuator Xinetics, Inc. deformable mirror to correct lower spatial frequency errors, and one or more of our existing 80x80 pixel (EEV39) low noise wavefront sensor cameras developed by SciMeasure Analytical Systems, Inc. Significant additional heritage from the sophisticated telemetry and software implementation of PALAO would also contribute to this effort. This testbed could be used to investigate issues related to multiple deformable mirror control, and for specific test cases (with sufficiently bright natural guide stars), multiconjugate wavefront sensing and control.

In addition to the engineering motivation for the development of this testbed, this system would also provide unprecedented Strehl ratio imaging of bright object at near-infrared wavelengths. This has compelling implications, for example, in the search of hot companions around nearby stars ${ }^{3}$. Because of the strong dependence of the guide star brightness requirement with wavelength, this system would have limited applicability to visible light science, due to limited sky coverage, but would include expanded science of bright objects, including several Solar System targets ${ }^{4}$.

\section{MULTICONJUGATE CORRECTION}

We propose a modification to the existing PALAO optical design to incorporate the woofer/tweeter mirror combination at different conjugate heights. We propose placing the 1600 element Photonex mirror, of active diameter $80 \mathrm{~mm}$, conjugate to the $5 \mathrm{~m}$ telescope primary, providing 41 actuators (40 subapertures) across the entrance pupil of the telescope. The 349 actuator mirror, placed conjugate to height altitude in the atmosphere, is illuminated by the same $80 \mathrm{~mm}$ beam, providing 11 actuators across the entrance pupil of the telescope, but providing off-axis correction across a significant field, up to 4 ' diameter, depending on the detailed location. Note that for the purpose of this paper, we shall hereafter consider the 349 actuator paper conjugate to a layer $6 \mathrm{~km}$ above the telescope.

With two deformable mirrors placed at different conjugate planes, the investigation of the practical limitations to multiconjugate adaptive optics (MCAO) will be possible. Unlike the recently proposed MCAO system for the Gemini South Telescope ${ }^{5}$, this MCAO testbed would be designed to allow rapid reconfiguration to explore alternative sensing and control approaches. Furthermore, because of the unprecedented spatial frequency control, these investigations can be conducted at visible, as well as near-infrared wavelengths, allowing the investigation of fields many regular isoplanatic angles in width.

Although the detailed design of the optimum conjugate location for each deformable mirror remains as future work, we consider the parameters presented in Table 1, which shows one possible allocation of conjugate mirrors.

\begin{tabular}{|l|r|r|r|r|r|}
\hline Deformable mirror & $\begin{array}{c}\text { Physical } \\
\text { actuator spacing } \\
(\mathrm{mm})\end{array}$ & $\begin{array}{c}\text { Active mirror } \\
\text { diameter } \\
(\mathrm{mm})\end{array}$ & $\begin{array}{c}\text { Conjugate height } \\
(\mathrm{km})\end{array}$ & $\begin{array}{c}\text { Projected } \\
\text { diameter at 10km } \\
(\boldsymbol{m})\end{array}$ & $\begin{array}{c}\text { Projected } \\
\text { actuator spacing } \\
(\boldsymbol{m})\end{array}$ \\
\hline 1600 actuator & 2 & 80 & 0 & 5.00 & 0.122 \\
\hline 349 actuator & 7 & 140 & 6 & 8.54 & 0.417 \\
\hline
\end{tabular}

Table 1. Possible MCAO system parameters used in this discussion. Detailed optimization of the MCAO system design remains as future work.

In reality, within the space of the $5 \mathrm{~m}$ telescope's $\mathrm{F} / 15.7$ focus, a $6 \mathrm{~km}$ layer is conjugate to a location that would occur before a recollimator providing an $80 \mathrm{~mm}$ pupil diameter. Thus the projected values for the 349 actuator mirror should be considered example values only. We note, however, that the pupil diameter at the 1600 actuator mirror represents a factor of $\sim 60$ demagnification, which is less than than currently invoked, for example, in the Keck AO systems. Furthermore, due to the $>40 \%$ reduction in beam diameter, the overall volume of this testbed would likely be reduced by over a factor of 3 compared to the existing PALAO system. 


\section{BRIGHT GUIDE STAR PERFORMANCE}

To estimate the potential of this system to achieving diffraction-limited visible and very high Strehl ratio near-infrared wavefront control, we present a performance prediction for this system in a variety of atmospheric conditions in Table 2 .

\begin{tabular}{|c|c|c|c|c|c|c|c|}
\hline $\begin{array}{l}\text { Atmospheric } \\
\text { Conditions }\end{array}$ & $\begin{array}{l}t_{0}[\mathrm{~cm} @ 0.5 \mu \mathrm{m}] \\
\text { Wind speed }[\mathrm{m} / \mathrm{s}] \\
\text { Seeing [arcseconds @ } 0.5 \mu \mathrm{m} \text { ] }\end{array}$ & $\begin{array}{c}7 \\
10 \\
1.5\end{array}$ & $\begin{array}{c}7 \\
5 \\
1.5\end{array}$ & $\begin{array}{c}10 \\
10 \\
1.0\end{array}$ & $\begin{array}{c}10 \\
5 \\
1.0\end{array}$ & $\begin{array}{c}15 \\
5 \\
0.69\end{array}$ & $\begin{array}{c}20 \\
5 \\
0.52\end{array}$ \\
\hline \multirow{5}{*}{ Error Terms } & $\sigma_{\mathrm{TD}}[\mathrm{nm}]$ & 113 & 63 & 84 & 47 & 34 & 26 \\
\hline & $\sigma_{\mathrm{RT}}[\mathrm{nm}]$ & 47 & 24 & 35 & 18 & 13 & 10 \\
\hline & $\sigma_{\mathrm{AF}}[\mathrm{nm}]$ & 67 & 67 & 50 & 50 & 35 & 28 \\
\hline & $\sigma_{\mathrm{TF}}[\mathrm{nm}]$ & 48 & 48 & 48 & 48 & 48 & 48 \\
\hline & $\sigma_{\mathrm{CAL}}[\mathrm{nm}]$ & 40 & 40 & 40 & 40 & 40 & 40 \\
\hline \multirow{5}{*}{$\begin{array}{l}\text { Predicted } \\
\text { Performance }\end{array}$} & $\sigma_{\text {TOTAL }}[n m]$ & 153 & 114 & 121 & 94 & 80 & 74 \\
\hline & Strehl Ratio @2.20 $\mu \mathrm{m}$ & 0.83 & 0.90 & 0.89 & 0.93 & 0.95 & 0.96 \\
\hline & Strehl Ratio @ $1.25 \mu \mathrm{m}$ & 0.55 & 0.72 & 0.69 & 0.80 & 0.85 & 0.87 \\
\hline & Strehl Ratio @ $0.90 \mu \mathrm{m}$ & 0.32 & 0.53 & 0.49 & 0.65 & 0.73 & 0.77 \\
\hline & Strehl Ratio@0.70 $\mu \mathrm{m}$ & 0.15 & 0.35 & 0.31 & 0.49 & 0.59 & 0.64 \\
\hline
\end{tabular}

Table 2. Predicted on-axis guide star performance, asumming $15 \mathrm{~Hz}$ tip/tilt and $40 \mathrm{~Hz}$ high order closed loop bandwidth, for 6 different atmospheric conditions. The error terms are TD (time delay), RT (residual tilt), AF (atmospheric fitting), TF (telescope fitting), and CAL (PSF calibration) errors. For detailed description of these terms, see Troy ${ }^{6}$. The final column represents good seeing conditions at Palomar Mountain.

As expected, the primary benefit of the 1600 actuator system is a significant reduction in the magnitude of the fitting error. Because fitting error for a continuous facesheet deformable mirror can be represented by,

$$
\sigma_{A F}^{2}=0.28\left(\frac{d}{r_{0}}\right)^{(5 / 3)}\left[\mathrm{rad}^{2}\right]
$$

where $\sigma_{A F}^{2}$ is the wavefront variance in radians squared, $\mathrm{d}$ is the actuator spacing as projected onto the sky, and $\mathrm{r} 0$ is the coherence parameter at the wavelength of consideration. The contribution at this term is reduced by a factor of $(40 / 15)^{\wedge} 5 / 3$ or approximately a factor of 5 when going from the existing PALAO d $=31.25 \mathrm{~cm}$ to the proposed $\mathrm{d}=12.5$ $\mathrm{cm}$. From Table 2, we see that for good seeing conditions (the rightmost column), which occur occasionally in summer months at Palomar Mountain, this system will be capable of $\mathrm{K}$-band Strehl ratios in excess of 95\%, which will scientifically exploit the strong dependancy of companion detection limit upon Strehl' ${ }^{7}$.

\subsection{Measurement Error}

Because of the small interactuator spacing, this 1600 actuator system would pay a penalty against its general use due to the increased requirement on guide star brightness. The wavefront error contribution attributable to measurement error for the proposed testbed is shown in Figure 1. 


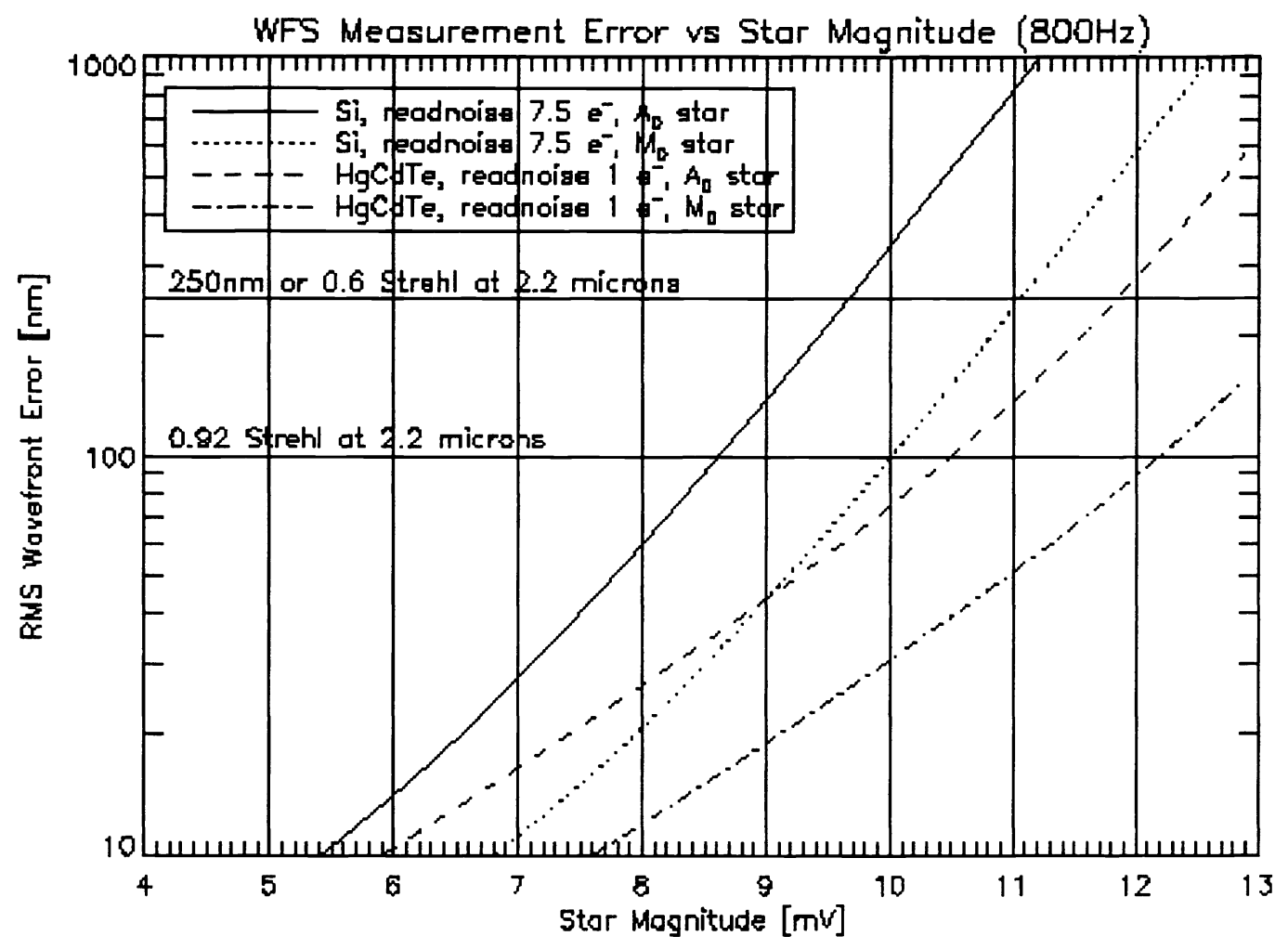

Figure 1. Measurement error for 1600 actuator Palomar MCAO testbed, assuming current technology $\mathrm{Si}$ detectors (visible wavefront sensing), and future low-noise $\mathrm{HgCdTe}$ detectors (sensing in visible and near infrared), for two limiting color natural guide stars, A0 and M0.

We find that, for $\mathrm{K}$-band operation, the small subaperture size still allows guiding on $\mathrm{mV}=9-10$ magnitude guide stars, at $800 \mathrm{~Hz}$ frame rates, with current detector technology. This limit is acceptable for an important class of scientific observation, namely the search for and scientific characterization of low-mass companions to nearby stars ${ }^{8}$. Note that in Figure 1, we also include the performance curves for a very promising, but yet-to-be-proven, low-noise, fast-frame-rate HgCdTe detector technology under development at Rockwell Science Center?.

\section{PHOTONEX MIRRORS}

Xinetics, Inc. has developed a new process for the fabrication of very high actuator count deformable mirrors. Using a proprietary multilayer ceramic structure that is cast, cofired, and finally precision sawed to provide dense actuator spacings, on the order of 1-4 mm in pitch. A 1600 actuator mirror, based upon this technology, has been fabricated by Xinetics. This prototype device possesses a $2 \mathrm{~mm}$ actuator pitch, and has been measured to provide $2 \mu \mathrm{m}$ of surface stroke using a command voltage in the range of 0-30 Volts. The static figure error of the facesheet has been measured at better than $\lambda / 20 \mathrm{rms}(0.633 \mu \mathrm{m})$ across $90 \%$ of the active area. This technology, which Xinetics markets as Photonex mirrors, promises to be scalable to over 7000 actuators where electrical connectivity issues dominate the fabrication process.

\section{SCIMEASURE CAMERAS}

To support multiple guide star experiments with the 1600 actuator upgrade, and in order to meet the future wavefront sensor needs for multiconjugate AO systems, SciMeasure is developing a modular CCD controller design that can control multiple CCDs from a single controller. This controller is based on an open bus architecture that allows for customization for any particular CCD or combination of similar CCDs. Each CCD can be provided with the required clock driver board, bias board, A/D boards and demultiplexer boards. These are all controlled from a single sequencer board and can utilize a 
common fiber optic link for remote operation. The bus has a bandwidth of 50 million 16-bit clock patterns per second and 50 million 16-bit pixels per second. For example, 16 ports could be read out at 3 million pix/sec/port. These 16 ports could be on a single CCD or more commonly, four 4-port CCDs such as the EEV CCD39 or the MIT/LL CCIDs.

In the case of multiple CCDs, provision is also made for varying the integration times in integral numbers of fundamental frame times. If multiple stars were to have different magnitudes, the readout frame rate for each CCD can be adjusted for the appropriate integration time. This is vastly superior to simply using a single larger CCD with a composite optical image of multiple stars.

This new modular architecture is presently being designed and is based on the proven design and layout which achieves noise performance of $7.5 \mathrm{rms}$ electrons at $2.1 \mathrm{million}$ pix/sec/port with the EEV CCD39, running in its own thermoelectrically cooled package at $\sim 25$ degC. Four EEV CCD39s configured in separate remote heads would yield an effective $160 \times 160$ pixel field of view sampled at 850 frames per second.

\section{WAVEFRONT PROCESSOR DESIGN}

One of the difficult problems with deformable mirror systems with a large number of actuators is the computer performance requirements. The reconstruction calculation, for example, for a Shack-Hartmann wavefront sensing scheme increases due to the need to interpret more subapertures of data and to control more actuators. In addition, as the fitting error terms reduce, the wavefront processor must provide higher rate closed loop performance in order to adequately reduce the temporal bandwidth error.

The current PALAO wavefront processor uses a matrix multiplication approach to reconstruct the wavefront. We are currently studying the advantages that using an FFT approach to reconstruct the wavefront would have for a 1600 actuator system. It is clear that an FFT approach would involve fewer number of numerical operations, however it is unclear that this would result in a system with better overall closed loop performance for this scale system. The detailed investigation of the impact of various reconstruction approaches on the phase margin of the control system remains as future work.

\section{ACKNOWLEDGMENTS}

Observations at the Palomar Observatory were made as part of a continuing collaborative agreement between Palomar Observatory, the Jet Propulsion Laboratory, and Cornell University.

\section{REFERENCES}

1. Raggazzoni, R., Marchetti, E., and Valente, G., "Adaptive optics corrections available for the whole sky", Nature, 403, 54-56 (2000)

2. Dekany, R., Nelson, J. E., and Bauman, B., "Design considerations for CELT adaptive optics", Proc. SPIE, 4003, (2000).

3. Oppenheimer, B. R., Kulkarni, S. R., Matthew, K., and van Kerkwijk, M. H., "The spectrum of the Brown Dwarf Gliese 229B", Astrophys. J., 502, 932-943, (1998).

4. Dekany, R., Banfield, D, Bouchez, A., Oppenheimer, B. R., Brown, M., Hayward, T., Brandl, B., Troy, M., Brack, G., Trinh, T., and Shi, F., "Solar system science with subarcsecond slit spectroscopy", Proc. SPIE, 4007, (2000).

5. Ellerbroek, B., private communication, Dec. 1999.

6. Troy, M., Dekany, R., Brack, G., Shi, F., Oppenheimer, B. R., Trinh, T., Bloemhof, E., Hayward, T., Brandl, B., and Dekens, F., "Palomar adaptive optics project: status and performance", Proc. SPIE, 4007, (2000).

7. Racine, R., Walker, G. A. H., Nadeau, D., Doyon, R., Marois, C., "Speckle noise and the detection of faint companions", PASP, 111, 587-594, (1999).

8. Dekany, R., "High-contrast observation potential of the Palomar Adaptive Optics System (PALAO)", in Proc. ESO Conference on Astronomical Results with Adaptive Optics, Sonthofen, Germany, in press, (1999).

9. Vural, K., private communication, March 2000. 